\title{
Erythropoietin ameliorates renal interstitial fibrosis via the inhibition of fibrocyte accumulation
}

\author{
XU CHANG GENG ${ }^{1 *}$, ZHOU PANG HU ${ }^{2 *}$ and GUO YONG LIAN ${ }^{1}$ \\ ${ }^{1}$ Department of Urology, Wuhan Central Hospital, Wuhan, Hubei 430014; \\ ${ }^{2}$ Department of Orthopedics, Renmin Hospital of Wuhan University, Wuhan, Hubei 430060, P.R. China
}

Received February 25, 2014; Accepted November 19, 2014

DOI: $10.3892 / \mathrm{mmr} .2015 .3157$

\begin{abstract}
Erythropoietin (EPO) is a hematopoietic hormone that protects against renal interstitial fibrosis in animal models; however, the mechanism underlying the anti-fibrotic activity of EPO has remained elusive. The present study aimed to elucidate this mechanism. Twenty-four male C57BL6 mice were randomly divided into four groups, each comprising six mice: i) control group (Sh); ii) unilateral ureteral obstruction (UUO) plus vehicle group (U+V); iii) UUO plus $300 \mathrm{U} / \mathrm{kg}$ body weight recombinant human (rh)EPO (U+E1) and iv) UUO plus $1,000 \mathrm{U} / \mathrm{kg}$ body weight rhEPO (U+E2). Seven days post-surgery, the mice were sacrificed for examination. UUO induced significant deposition of extracellular matrix, detected by picro-sirius red staining, which was decreased following rhEPO treatment. UUO also induced deposition of collagen I and fibronectin, rhEPO treatment was able to attenuate this effect at protein and mRNA levels. Compared with the control groups, UUO resulted in the accumulation of $\alpha$-smooth muscle actin-positive cells in the interstitium, an effect which was ameliorated by rhEPO. Furthermore, rhEPO abrogated the UUO-induced increase in the number of bone marrow-derived myofibroblasts. Mechanistically, it was discovered that rhEPO decreased CXC chemokine ligand 16 (CXCL16) expression at protein level. However, treatment with rhEPO did not alter the protein expression of CC chemokine ligand 21 or CXCL12. These results suggested that rhEPO decreased fibrocyte accumulation via the suppression of renal CXCL16, which resulted in the attenuation of renal fibrosis.
\end{abstract}

Correspondence to: Dr Guo Yong Lian, Department of Urology, Wuhan Central Hospital, 26 Shengli Street, Wuhan, Hubei 430014, P.R. China

E-mail: guoyonglianurology@126.com

*Contributed equally

Key words: erythropoietin, renal fibrosis, fibrocyte, chemokine

\section{Introduction}

Chronic kidney disease induces irreversible kidney damage, and progressive renal fibrosis is frequently observed regardless of the underlying cause of the disease (1). The major effector cells that are associated with the development of progressive renal fibrosis are the interstitial myofibroblasts (2). Interstitial myofibroblasts have been proposed to originate from five potential sources, including circulating fibrocytes, pericytes, fibroblasts, the tubular epithelial-mesenchymal transition (EMT) and the endothelial-mesenchymal transition (3).

Fibrocytes are bone marrow-derived mesenchymal progenitor cells, which express hematopoietic stem cell antigens, monocytic lineage markers and fibroblast products (4). Fibrocytes constitutively produce extracellular matrix (ECM) components, in addition to ECM-modifying enzymes, and are able to differentiate into myofibroblasts in vitro and in vivo under certain micro-environmental conditions (5). There is an increasing body of evidence suggesting that these cells contribute to the development of the novel myofibroblast population that is responsible for the production of ECM during renal fibrosis (6-11).

Erythropoietin (EPO) is a hematopoietic hormone, the majority of which is produced by the adult kidneys. In addition to its erythropoietic effects, EPO exerts protective effects against acute ischemic and toxic renal injuries (12-14). EPO also protects against interstitial injuries, including interstitial fibrosis, in a variety of animal models of human disease (15-18). The inhibition of EMT is one potential mechanism underlying the anti-fibrotic effects of EPO $(16,18)$. However, whether the beneficial effects of EPO in renal fibrosis are associated with the inhibition of fibrocyte accumulation remains to be elucidated. The aim of the present study was to determine whether recombinant human (rh)EPO treatment was able to prevent the progression of renal fibrosis via the attenuation of interstitial fibrocytes in a mouse model of complete unilateral ureteral obstruction (UUO).

\section{Materials and methods}

Animals, agents and antibodies. Male C57BL6 mice (18-20 g) were purchased from the Experimental Animal Center of Wuhan University (Wuhan, China). Animals were housed in standard rodent cages with ad libitum access to water and food 
until sacrifice. All surgical and experimental procedures were approved by the Institutional Animal Care and Use Committee of Wuhan University (Wuhan, China). rhEPO was obtained from Sunshine Pharmaceutical Company (Shenyang, China). Rabbit polyclonal anti-human collagen I (cat. no. ab34710), Rabbit polyclonal anti-human fibronectin (cat. no. ab2413), Rabbit polyclonal anti-human $\alpha$ smooth muscle actin $(\alpha-\mathrm{SMA}$; cat.no. ab5694) and Rabbit polyclonal anti-mouse CXC chemokine ligand 16 (CXCL16; cat. no. ab119350) antibodies were purchased from Abcam (Cambridge, UK). Rabbit Polyclonal anti-mouse CXCL12 (cat. no. PA1-29029) was purchased from Thermo Fisher Scientific (Waltham, MA, USA). Goat Polyclonal anti-mouse CC chemokine ligand 21 (CCL21) antibody was purchased from R\&D Systems, Inc. (cat. no. AF457; Minneapolis, MN, USA). Rabbit polyclonal anti-human actin (cat. no. sc-7210) and Rat monoclonal anti-mouse CD45 (cat. no. sc-21739) antibodies were purchased from Santa Cruz Biotechnology, Inc. (Dallas, TX, USA). Donkey monoclonal anti-rabbit or donkey anti-goat secondary antibodies were purchased from Rockland Immunochemicals, Inc. (Limerick, PA, USA).

Experimental protocol. Twenty-four male C57BL6 mice were randomly assigned into four groups, each comprising six mice: i) control group (Sh); ii) UUO plus vehicle group (U+V); iii) UUO plus $300 \mathrm{U} / \mathrm{kg}$ body weight rhEPO (U+E1) and iv) UUO plus $1,000 \mathrm{U} / \mathrm{kg}$ body body weight rhEPO (U+E2). UUO was performed as described previously (19). Briefly, under sodium pentobarbital $(60 \mathrm{mg} / \mathrm{kg}$ body weight; Merck, Darmstadt, Germany) anesthesia, the left ureter was exposed and completely ligated with 4-0 sutures, following a left abdominal incision. The sham-operation mice had their ureters exposed and manipulated, but not ligated. For rhEPO treatment, $300 \mathrm{U} / \mathrm{kg}$ or $1,000 \mathrm{U} / \mathrm{kg}$ body weight $\mathrm{rhEPO}$ was administered intraperitoneally daily from day one to day six following UUO. In the U+V group, vehicle (phosphate-buffered saline) was administered intraperitoneally following a protocol identical to the rhEPO treatment. Mice were sacrificed on the seventh day following surgery, and the obstructed kidneys were harvested. One third of the kidney was fixed in $4 \%$ buffered paraformaldehyde (Boshide, Wuhan, China) and embedded in paraffin for histological and immunohistochemical studies. One third of the kidney was stored for flow cytometric analysis, and the remaining kidney sections were snap-frozen in liquid nitrogen and stored at $-80^{\circ} \mathrm{C}$ for protein and RNA extraction.

Histological and immunohistochemical examination. Kidney sections $(5 \mu \mathrm{m})$ were prepared from the paraffin-embedded tissue specimens. Total collagen was identified using picro-sirius red (PSR) staining following a previously described method with modifications (20). For immunohistochemical examination, the renal sections were incubated with anti-collagen I antibody (1:100), anti-fibronectin antibody (1:100) or anti- $\alpha$-SMA antibody (1:200). The kidney specimens were incubated with the primary antibodies overnight at $4^{\circ} \mathrm{C}$, followed by a second reaction with anti-rabbit antibody conjugated with envision polymer (Thermo Fisher Scientific) for $30 \mathrm{~min}$. Finally, a diaminobenzidine reaction was performed on the section using a kit (TL-015-QHD; Thermo
Fisher Scientific) and hematoxylin (Boshide) was used as the counterstain.

Western blot analysis. Western blot analysis was performed as previousy described (21). In brief, protein samples were heated at $100^{\circ} \mathrm{C}$ for $5 \mathrm{~min}$ and subjected to electrophoresis on 10 or $15 \%$ SDS gels. Proteins were electrophoretically transferred to nitrocellulose membranes (Millipore, Billerica, MA, USA) which were subsequently incubated with antibodies specific for $\alpha$-SMA $(1: 500)$, collagen I $(1: 1,000)$, fibronectin (1:400), CXCL12 (1:1,000), CCL21 (1:600), CXCL16 (1:500) and $\beta$-actin $(1: 1,000)$, followed by incubation with secondary antibody conjugated with IRDye ${ }^{\circledR}$ infrared dye (Rockland Immunochemicals, Inc.). The signals were detected using an Odyssey Infrared Imaging System (Li-COR Biosciences, Lincoln, NE, USA). Actin was used as an internal control.

Reverse transcription quantitative polymerase chain reaction (RT-qPCR). qPCR, following reverse transcription was used to assess transcript levels of collagen I and fibronectin. RNA was extracted from frozen tissue by homogenization in TRIzol ${ }^{\circledR}$ (Invitrogen Life Technologies, Carlsbad, CA, USA), and $1 \mu \mathrm{g}$ aliquots of RNA were used in a reverse transcription reaction with $\mathrm{M}-\mathrm{MuLV}$ reverse transcriptase (cat. no. K1621; Thermo Fisher Scientific). The resulting complementary DNA was used as a template for qPCR analysis. Primers were obtained from Sangon Biological Engineering Technology and Services (Shanghai, China), and the specific primers were designed as follows: Forward, 5'-TGCCGC GACCTCAAGATGTG-3' and reverse, 5'-CACAAGGGT GCTGTAGGTGA-3' for collagen I; forward 5'-CTTCTC CGTGGAGTTTTACCG-3' and reverse, 5'GCTGTCAAA TTGAATGGTGGTG-3' for fibronectin and forward, 5'-GGT GAAGGTCGGTGTGAACG-3' and reverse, 5'-CTCGCT CCTGGAAGATGGTG-3' for GAPDH. A $20 \mu 1$ sample of PCR reaction solution, which included SYBR Green PCR Master Mix (\#RR420A; TaKaRa Bio, Inc., Otsu, Japan), was amplified according to the manufacturer's instructions. Quantifications were performed in duplicate on the ABI Prism 7500 Sequence Detection System (Applied Biosystems Life Technologies, Foster City, CA, USA). The calculations of the relative change in mRNA were performed using the ${ }^{\Delta \Delta} \mathrm{Ct}$ method.

Flow cytometry. Renal cells were isolated as previously described with modifications (22). Briefly, the kidneys were decapsulated, minced finely and incubated at $37^{\circ} \mathrm{C}$ for $40 \mathrm{~min}$ in phosphate-buffered saline (Boshide) containing $0.25 \%$ trypsin (Biyuntian, Wuhan, China). Cells were filtered through a 40- $\mu \mathrm{m}$ strainer, rinsed, centrifuged and resuspended in fluorescence activated cell sorting buffer (Boshide). Cells $\left(5 \times 10^{5}\right)$ were incubated with anti-CD45 and anti-collagen I antibodies. Subsequently, the cells were incubated with fluorescein isothiocyanate/phycoerythrin-labeled secondary antibodies (Santa Cruz Biotechnology, Inc.). Certain cells were incubated with irrelevant isotype-matched antibodies (Santa Cruz Biotechnology, Inc.) and unstained cells were used as controls. The cut-off values were determined according to the results of the control experiments. Data were analyzed using BD FACSDiva ${ }^{\mathrm{TM}}$ software (BD Biosciences, Franklin Lakes, NJ, USA). 


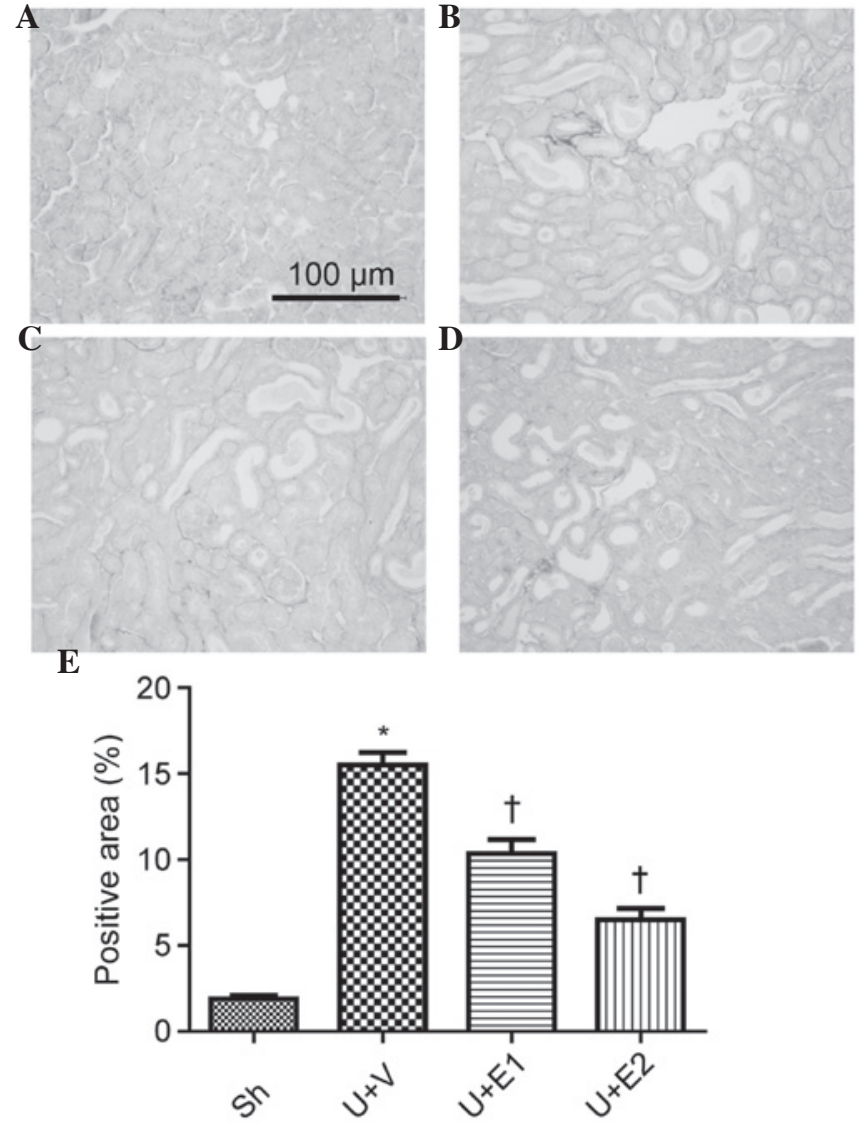

Figure 1. Renal interstitial fibrosis was examined by picrio-sirus red staining on day seven post-surgery. (A) Sh group, (B) U+V group, (C) U+E1 group, (D) U+E2 group. (E) Semi-quantitative assessment of renal fibrosis. Values are expressed and the mean \pm standard deviation. ${ }^{*} \mathrm{P}<0.05$ vs. the Sh group; ${ }^{\dagger} \mathrm{P}<0.05$ vs. U+V group. UUO, unilateral ureteral obstruction; Sh, control; $\mathrm{U}+\mathrm{V}$, UUO+vehicle; U+E1, UUO treated with $300 \mathrm{U} / \mathrm{kg}$ rhEPO; rhEPO, recombinant human erythropoietin; U+E2, UUO treated with $1,000 \mathrm{U} / \mathrm{kg}$ rhEPO.

Statistical analysis. Statistical analyses of the data were performed using the Graphpad Prism ${ }^{\circledR}$ software package, version 5.0 (Graphpad Software, Inc., La Jolla, CA, USA) Values are expressed as the mean \pm standard deviation. Multiple group comparisons were performed by one-way analysis of variance followed by the Bonferroni procedure for the comparison of means. $\mathrm{P}<0.05$ was considered to indicate a statistically significant difference between values.

\section{Results}

EPO treatment suppresses renal tubulo-interstitial fibrosis. UUO induced an increase in collagen I expression on the PSR-stained kidney sections, in comparison to that of the control group on day seven. By contrast, collagen levels were reduced in the rhEPO-treated UUO kidneys, compared with those of the vehicle treated UUO kidneys (Fig. 1). This was verified by semi-quantitative evaluation of the fibrotic area of the PSR-stained kidney sections. Furthermore, compared with the control and vehicle-treated UUO kidneys, rhEPO-treatment suppressed renal collagen I and fibronectin expression at the mRNA and protein levels (Fig. 2). These results indicated an anti-fibrotic effect of rhEPO.
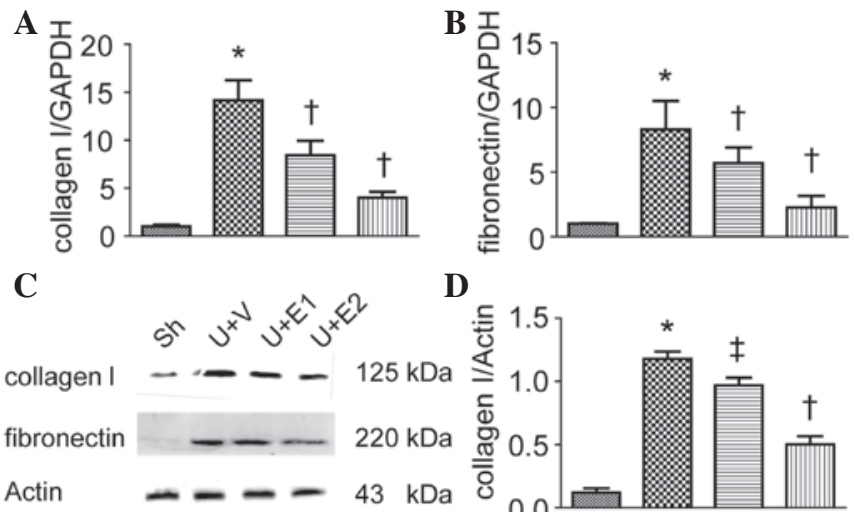

D

E

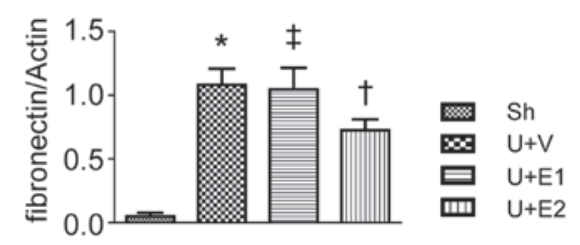

Figure 2. Semi-quantitative analysis of mRNA expression levels of (A) collagen I and (B) fibronectin. (C) Representative gels of the western blot analysis of collagen and fibronectin protein expression levels. Semi-quantitative analysis of (D) collagen I and (E) fibronectin protein expression. Actin was used as an internal control. Values are expressed as the mean \pm standard deviation. ${ }^{*} \mathrm{P}<0.05$ vs. Sh; ${ }^{\dagger} \mathrm{P}<0.05$ vs. U+V and ${ }^{\ddagger} \mathrm{P}>0.05$ vs. U+V. UUO, unilateral ureteral obstruction; Sh, control; U+V, UUO+vehicle; U+E1, UUO treated with $300 \mathrm{U} / \mathrm{kg}$ rhEPO; rhEPO, recombinant human erythropoietin; U+E2, UUO treated with $1,000 \mathrm{U} / \mathrm{kg}$ rhEPO.

EPO treatment attenuates myofibroblast accumulation. Myofibroblasts are the effector cells for ECM production; therefore, whether EPO treatment decreased myofibroblast accumulation was evaluated. The control kidneys revealed an absence of or weak labeling of $\alpha$-SMA (Fig. 3A), which surrounded the major blood vessels, whereas the vehicle-treated UUO kidneys demonstrated increased expression of $\alpha$-SMA in the interstitium (Fig. 3B). However, rhEPO-treatment (groups U+E1 and U+E2) attentuated the accumulation of $\alpha$-SMA positive cells (Fig. 3C and D). Consistent with the results of the immunohistochemical analysis, immunoblotting revealed an increase in $\alpha$-SMA expression levels in the obstructed kidneys of the vehicle-treated UUO mice in comparison to those of the control group. By contrast, rhEPO treatment significantly inhibited $\alpha$-SMA upregulation (Fig. 3E and F).

EPO treatment decreases fibrocyte accumulation in the interstitium. Myofibroblasts are potentially derived from multiple sources, so whether rhEPO treatment was able to decrease fibrocyte accumulation was investigated. Compared with the control group, UUO increased fibrocyte accumulation in the interstitium, as detected by flow cytometry (Fig. 4Aa, Ab and B). By contrast, rhEPO (U+E1 and U+E2 groups) treatment significantly inhibited the accumulation of fibrocytes (Fig. 4Ac, Ad and B).

EPO decreases CXCL16 expression. Bone marrow-derived fibrocytes migrate into the kidney by following chemoattractants, including CXCL12, CCL21 and CXCL16. Whether 


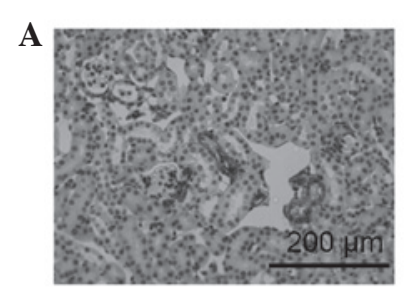

$\mathbf{E}$

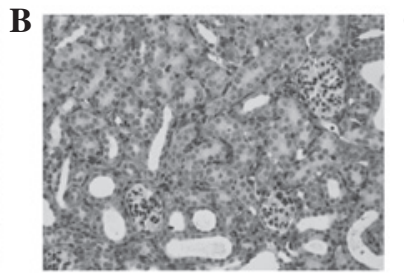

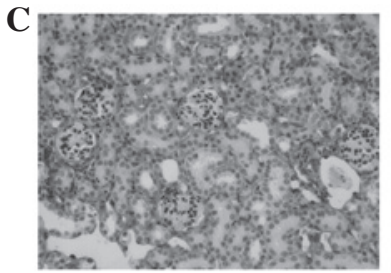

F
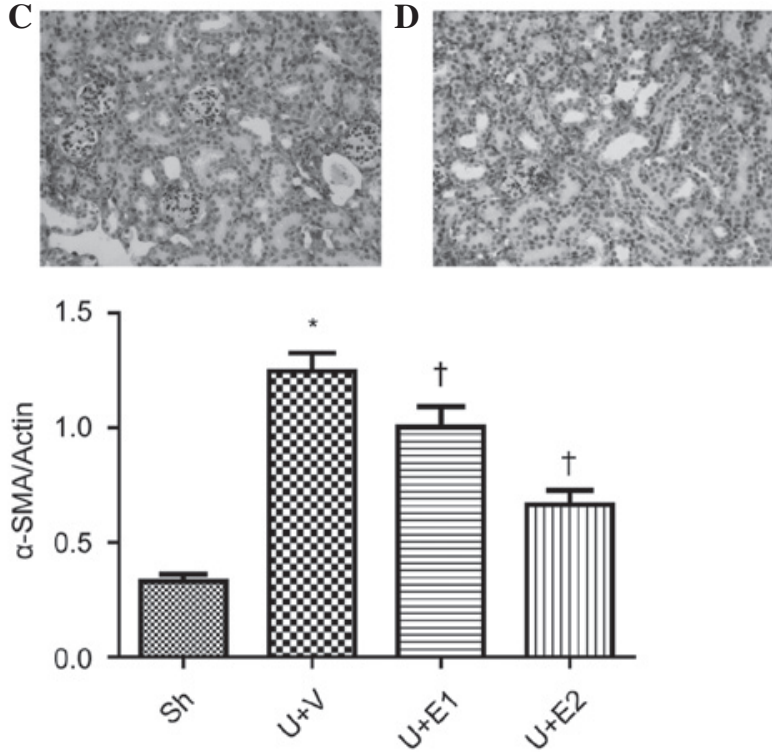

Figure 3. Representative photographs of $\alpha$-SMA immunohistochemistry on day 7 post-surgery. (A) Sh, (B) U+V, (C) U+E1 and (D) U+E2 groups. (E) Representative gels of the western blot analysis of $\alpha$-SMA expression levels. (F) Semi-quantitative analysis of $\alpha$-SMA protein expression. Actin was used as an internal control. "P<0.05 vs. Sh; ${ }^{\dagger} \mathrm{P}<0.05$ vs. U+V. Values are expressed as the mean \pm standard deviation. Scale bar, $200 \mu \mathrm{m}$. UUO, unilateral ureteral obstruction; Sh, control; U+V, UUO+vehicle; U+E1, UUO treated with $300 \mathrm{U} / \mathrm{kg}$ rhEPO; rhEPO, recombinant human erythropoietin; U+E2, UUO treated with $1,000 \mathrm{U} / \mathrm{kg}$ rhEPO; $\alpha$-SMA, $\alpha$-smooth muscle actin.

A
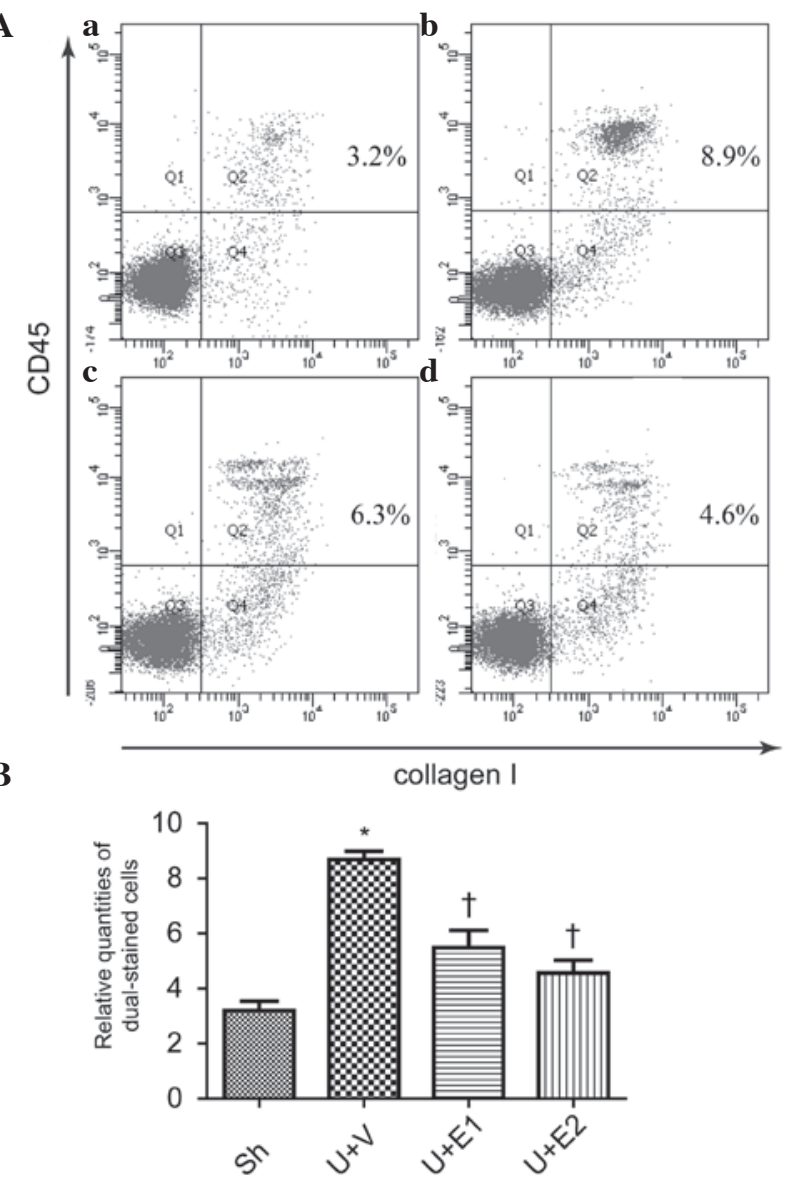

Figure 4. Fibrocytes in the interstitium were detected by flow cytometry, indicated by the percentage of cells positively dual-stained for collagen I and CD45. (A) Representative photographs of (a) Sh, (b) U+V, (c) U+E1 and (d) U+E2. (B) Semi-quantitative analysis of the percentage of dual-stained cells. ${ }^{*} \mathrm{P}<0.05$ vs. Sh; ${ }^{\dagger} \mathrm{P}<0.05$ vs. U+V. UUO, unilateral ureteral obstruction; Sh, control; U+V, UUO+vehicle; U+E1, UUO treated with $300 \mathrm{U} / \mathrm{kg}$ rhEPO; rhEPO, recombinant human erythropoietin; U+E2, UUO treated with $1,000 \mathrm{U} / \mathrm{kg}$ rhEPO.
A
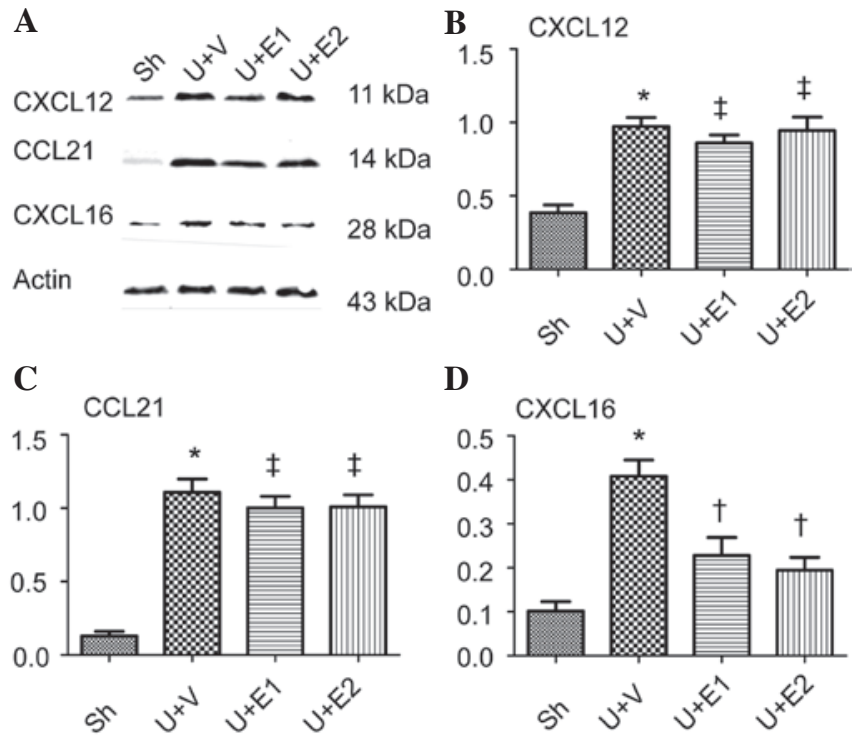

$\mathbf{D}$

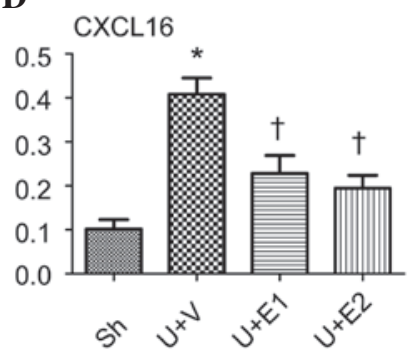

Figure 5. CXCL12, CCL21 and CXCL16 expression analysis. (A) Representative western blots for CXCL12, CCL21 and CXCL16. Semi-quantitative analysis of protein expression levels of (B) CXCL12, (C) CCL21 and (D) CXCL16. Actin was used as an internal control. ${ }^{*} \mathrm{P}<0.05$ vs. Sh; ${ }^{\dagger} \mathrm{P}<0.05$ vs. U+V and ${ }^{\star} \mathrm{P}>0.05$ vs. U+V. UUO, unilateral ureteral obstruction; Sh, control; U+V, UUO+vehicle; U+E1, UUO treated with $300 \mathrm{U} / \mathrm{kg}$ rhEPO; rhEPO, recombinant human erythropoietin; U+E2, UUO treated with 1,000 U/kg rhEPO; CXCL16, CXC chemokine ligand 16; CCL21, CC chemokine ligand 21.

rhEPO treatment was able to alter the expression of these chemokines was examined. Compared with the control group, increases in the expression of CXCL12, CCL21 and CXCL16 were observed following UUO. However, rhEPO treatment (U+E1 and $\mathrm{U}+\mathrm{E} 2$ groups) decreased the expression of CXCL16 but did not influence CXCL12 or CCL21 expression (Fig. 5). 


\section{Discussion}

At present, no specific therapeutic modalities for chronic renal disease are available (23). However, the prevention or inhibition of renal fibrosis is one therapeutic option that may potentially be capable of slowing, or even stopping the progression of chronic renal disease. In the present study, it was demonstrated that rhEPO ameliorated renal fibrosis in mice following complete UUO. Furthermore, rhEPO decreased the expression of CXCL16 and the accumulation of fibrocytes in the obstructed kidneys. Therefore, in addition to its function in the treatment of anemia, the results of the present study suggested that rhEPO treatment may be used in the inhibition of renal fibrosis, in part via the attenuation of fibrocyte accumulation in the kidneys.

In order to analyze the anti-fibrotic effects of rhEPO, a UUO mouse model was used. The UUO mouse model is a well established animal model for the induction of renal fibrosis and assessment of the anti-fibrotic effects of various agents. The model remains free of confounding factors, including exogenous toxins or uremia, as the unobstructed kidney maintains normal homeostasis and renal function (19). ECM was examined using PSR staining, which indicated rhEPO decreased ECM accumulation. This result was corroborated by PCR and western blot analyses of collagen I and fibronectin expression. These results suggested an anti-fibrotic effect of rhEPO.

The $\alpha$-SMA positive cells are the main effectors contributing to renal fibrosis (24). The results of the present study demonstrated that rhEPO treatment attenuated the accumulation of $\alpha$-SMA positive cells, which may partially explain the anti-fibrotic effects of rhEPO. However, the origin of the myofibroblasts responsible for the excessive production of ECM has remained elusive (25). A previous study demonstrated that EMT may be an essential process in renal fibrogenesis (26). Multiple studies have provided evidence that bone marrow-derived myofibroblasts may be recruited to the kidney and subsequently contribute to kidney fibrosis $(6-8,10,11,27,28)$.

Bone marrow-derived myofibroblast precursor cells, named 'fibrocytes', were initially identified in the peripheral circulation (29). These fibrocytes were found to co-express mesenchymal markers, including collagen I and vimentin, as well as hematopoietic markers, including CD45, CD11b and CD34 (30). In culture, fibrocytes exhibited an adherent, spindle-like morphology and expressed $\alpha$-SMA following treatment with TGF $\beta 1$, which was consistent with the hypothesis that these cells are able to differentiate into myofibroblasts (4). In the present study, bone marrow-derived myofibroblast precursors were demonstrated to accumulate in the kidney following obstructive injury. Flow cytometric analysis indicated that UUO induced CD45 and collagen I dual-positive myofibroblast precursors to migrate into the injured kidneys, an effect which was abrogated following rhEPO treatment. These results suggested that the anti-fibrotic effect of rhEPO may be partially due to the decreased accumulation of fibrocytes in the obstructed kidneys.

The signaling mechanisms underlying the recruitment of bone marrow-derived myofibroblast precursors into the kidney remain to be elucidated (31). Chemokines have significant roles in the regulation of myofibroblast precursor infiltration in response to injury. Chemokines are classified into four major families: C, CC, CXC and CX3C, based on the relative position of cysteine residues proximal to the amino terminus (31).
Chemokines activate seven-transmembrane G-protein-coupled receptors and have primary functions in the trafficking of circulating cells during inflammation.

Sakai et al (8) demonstrated that CCL21 and its receptor, CCR7, were involved in the infiltration of myofibroblast precursors into the kidney in a mouse model of renal fibrosis induced by obstructive injury. A further study identified a role for $\mathrm{CXC} 12$ in the accumulation of fibrocytes in animal models of injury-induced pulmonary fibrosis (32). In addition, Chen et al (9) revealed that the CXCL16-CXCR6 axis was associated with fibrocyte recruitment. The present study aimed to evaluate whether chemokines CXCL12, CXCL16 and CCL21 were engaged in fibrocyte recruitment. The results indicated that UUO induced upregulation of CXCL12, CXCL16 and CCL21, and rhEPO treament abrogated the upregulation of CXCL16, but did not influence the expression of CXCL12 and CCL12. These results suggested that rhEPO decreased renal CXCL16 expression, indicating that this is may be one of the mechanisms underlying the reduction of fibrocyte accumulation in the interstitium.

A low dosage of rhEPO decreased the mRNA expression levels of collagen I and fibronectin, but did not alter the protein expression levels. It was postulated that rhEPO influenced the post-transcriptional processes. In addition, it was demonstrated that a low dose of rhEPO ameliorated renal fibrosis, but did not decrease the number of $\alpha$-SMA-positive cells. This inconsistency may be due to the fact that $\alpha$-SMA positive cells are the main cells responsible for ECM production, but are not the only cells involved (33). The effects of long-term rhEPO treatment on fibrocyte accumulation remain to be elucidated and requires further investigation.

In conclusion, it was demonstrated that rhEPO attenuated fibrocyte accumulation in the interstitium, indicating that rhEPO may present a potential strategy for the prevention of interstitial fibrosis and slow the progression of chronic renal insufficiency. The model used in the present study indicated that rhEPO decreased CXCL16, a chemokine that attracts circulating fibroblast-like precursors, and attenuated the accumulation of bone marrow-derived myofibroblasts. To the best of our knowledge, this is the first study to report the beneficial effects of rhEPO treatment, beyond hematopoiesis, against renal fibrosis via the inhibition of fibrocyte accumulation. These results may therefore provide novel insights into the mechanisms underlying the protection conferred by treatment with rhEPO against chronic kidney disease.

\section{References}

1. Duffield JS: Cellular and molecular mechanisms in kidney fibrosis. J Clin Invest 124: 2299-2306, 2014

2. Boor P and Floege J: The renal (myo-) fibroblast: a heterogeneous group of cells. Nephrol Dial Transplant 27: 3027-3036, 2012.

3. Grgic I, Duffield JS and Humphreys BD: The origin of interstitial myofibroblasts in chronic kidney disease. Pediatr Nephrol 27: 183-193, 2012.

4. Bellini A and Mattoli S: The role of the fibrocyte, a bone marrow-derived mesenchymal progenitor, in reactive and reparative fibroses. Lab Invest 87: 858-870, 2007.

5. Pilling D and Gomer RH: Differentiation of circulating monocytes into fibroblast-like cells. Methods Mol Biol 904: 191-206, 2012.

6. Iwano M, Plieth D, Danoff TM, Xue C, Okada H and Neilson EG: Evidence that fibroblasts derive from epithelium during tissue fibrosis. J Clin Invest 110: 341-350, 2002. 
7. Li J, Deane JA, Campanale NV, Bertram JF and Ricardo SD: The contribution of bone marrow-derived cells to the development of renal interstitial fibrosis. Stem Cells 25: 697-706, 2007.

8. Sakai N, Wada T, Yokoyama H, et al: Secondary lymphoid tissue chemokine (SLC/CCL21)/CCR7 signaling regulates fibrocytes in renal fibrosis. Proc Natl Acad Sci USA 103: 14098-14103, 2006.

9. Chen G, Lin SC, Chen J, et al: CXCL16 recruits bone marrow-derived fibroblast precursors in renal fibrosis. J Am Soc Nephrol 22: 1876-1886, 2011

10. Reich B, Schmidbauer K, Rodriguez Gomez M, et al: Fibrocytes develop outside the kidney but contribute to renal fibrosis in a mouse model. Kidney Int 84: 78-89, 2013.

11. Yang J, Lin SC, Chen G, et al: Adiponectin promotes monocyte-to-fibroblast transition in renal fibrosis. J Am Soc Nephrol 24: 1644-1659, 2013.

12. Oba S, Suzuki E, Nishimatsu H, et al: Renoprotective effect of erythropoietin in ischemia/reperfusion injury: possible roles of the Akt/endothelial nitric oxide synthase-dependent pathway. Int J Urol 19: 248-255, 2012.

13. Kaynar K, Aliyazioglu R, Ersoz S, et al: Role of erythropoietin in prevention of amikacin-induced nephropathy. J Nephrol 25: 744-749, 2012.

14. Wang W and Zhang J: Protective effect of erythropoietin against aristolochic acid-induced apoptosis in renal tubular epithelial cells. Eur J Pharmacol 588: 135-140, 2008.

15. Imamura R, Isaka Y, Sandoval RM, et al: A nonerythropoietic derivative of erythropoietin inhibits tubulointerstitial fibrosis in remnant kidney. Clin Exp Nephrol 16: 852-862, 2012.

16. Chen CL, Chou KJ, Lee PT, et al: Erythropoietin suppresses epithelial to mesenchymal transition and intercepts Smad signal transduction through a MEK-dependent mechanism in pig kidney (LLC-PK1) cell lines. Exp Cell Res 316: 1109-1118, 2010.

17. Kitamura H, Isaka Y, Takabatake Y, et al: Nonerythropoietic derivative of erythropoietin protects against tubulointerstitial injury in a unilateral ureteral obstruction model. Nephrol Dial Transplant 23: 1521-1528, 2008.

18. Park SH, Choi MJ, Song IK, et al: Erythropoietin decreases renal fibrosis in mice with ureteral obstruction: role of inhibiting TGF-beta-induced epithelial-to-mesenchymal transition. J Am Soc Nephrol 18: 1497-1507, 2007.

19. Chevalier RL, Forbes MS and Thornhill BA: Ureteral obstruction as a model of renal interstitial fibrosis and obstructive nephropathy. Kidney Int 75: 1145-1152, 2009.
20. Grande MT, Fuentes-Calvo I, Are'valo M, et al: Deletion of H-Ras decreases renal fibrosis and myofibroblast activation following ureteral obstruction in mice. Kidney Int 77: 509-518, 2010.

21. Kim DH, Moon SO, Jung YJ, et al: Mast cells decrease renal fibrosis in unilateral ureteral obstruction. Kidney Int 75 : 1031-1038, 2009.

22. Li L, Huang L, Sung SS, et al: The chemokine receptors CCR2 and CX3CR1 mediate monocyte/macrophage trafficking in kidney ischemia-reperfusion injury. Kidney Int 74: 1526-1537, 2008.

23. Formentini I, Bobadilla M, Haefliger C, et al: Current drug development challenges in chronic kidney disease (CKD) - identification of individualized determinants of renal progression and premature cardiovascular disease (CVD). Nephrol Dial Transplant 27 Suppl 3: iii81-iii88, 2012.

24. Strutz F and Zeisberg M: Renal fibroblasts and myofibroblasts in chronic kidney disease. J Am Soc Nephrol 17: 2992-2998, 2006.

25. Meran S and Steadman R: Fibroblasts and myofibroblasts in renal fibrosis. Int J Exp Pathol 92: 158-167, 2011.

26. Kriz W, Kaissling B and Le Hir M: Epithelial-mesenchymal transition (EMT) in kidney fibrosis: fact or fantasy? J Clin Invest 121: 468-474, 2011.

27. Broekema M, Harmsen MC, van Luyn MJ, et al: Bone marrow-derived myofibroblasts contribute to the renal interstitial myofibroblast population and produce procollagen I after ischemia/reperfusion in rats. J Am Soc Nephrol 18: 165-175, 2007.

28. Niedermeier M, Reich B, Rodriguez Gomez M, et al: CD4+ $\mathrm{T}$ cells control the differentiation of Gr1+ monocytes into fibrocytes. Proc Natl Acad Sci USA 106: 17892-17897, 2009.

29. Bucala R, Spiegel LA, Chesney J, Hogan M and Cerami A: Circulating fibrocytes define a new leukocyte subpopulation that mediates tissue repair. Mol Med 1: 71-81, 1994.

30. Wada T, Sakai N, Matsushima K and Kaneko S: Fibrocytes: a new insight into kidney fibrosis. Kidney Int 72: 269-273, 2007.

31. Chung AC and Lan HY: Chemokines in renal injury. J Am Soc Nephrol 22: 802-809, 2011.

32. Phillips RJ, Burdick MD, Hong K, et al: Circulating fibrocytes traffic to the lungs in response to CXCL12 and mediate fibrosis. J Clin Invest 114: 438-446, 2004.

33. Boor P, Ostendorf T and Floege J: Renal fibrosis: novel insights into mechanisms and therapeutic targets. Nat Rev Nephrol 6: 643-656, 2010. 